\title{
Isolation of Glucoamylase-non-producing Mutants in the Yeast Saccharomyces diastaticus
}

\author{
Ichiro YAmASHITA and Sakuzo FUKUI \\ Department of Fermentation Technology, \\ Faculty of Engineering, Hiroshima University, \\ Shitami, Saijo-cho, Higashi-Hiroshima 724, Japan
}

Received June 27, 1983

\begin{abstract}
Saccharomyces diastaticus produces extracellular glucoamylases and ferments starch. We have isolated glucoamylase-non-producing (amy) mutants from wild-type strains of $S$. diastaticus carrying a $S T A 1$ gene after mutagenesis with ethylmethane sulfonate. All amy mutations were recessive and were divided into two complementation groups, amy1 and amy2. The amy1 group may be located in the $S T A 1$ gene. The amy 2 mutation was involved in both glucoamylase production and flocculation.
\end{abstract}

Saccharomyces diastaticus carrying any one of the unlinked $S T A$ genes $S T A 1, S T A 2$, or $S T A 3$, secretes glucoamylase-isozymes I, II, or III, respectively, in the culture medium and ferments starch. ${ }^{1,2)}$ The secreted enzymes are glycosylated. ${ }^{2)}$ We have been interested in the genetic and biochemical control of synthesis and secretion of the glycosylated enzymes ${ }^{3}$ and have isolated glucoamylase-nonproducing (amy) mutants from $S$. diastaticus strains carrying the $S T A 1$ gene. In this paper, we give a genetic analysis of the amy mutants. $S$. diastaticus strains used and their relevant genotypes are listed in Table I. The strains are highly flocculent since they carry a dominant flocculation gene $F L O 8$, a novel flocculation gene derived from a $S$. diastaticus strain 510632D, kindly provided by $\mathrm{H}$. Tamaki, and the details of the gene will be published elsewhere (manuscript No. 3-355).

\section{MATERIALS AND METHODS}

Strains. Yeast strains used and their relevant genotypes are listed in Table I.

Culture media. YPS medium contained $1 \%$ yeast extract, $2 \%$ peptone, and $3 \%$ soluble starch. YPSB medium was YPS medium containing $3.3 \times 10^{-3 \%}$ bromocresol purple. YEPD medium contained $1 \%$ yeast extract, $2 \%$ peptone, and $2 \%$ dextrose. If necessary, the media were solidified by $2 \%$ agar.

Fermentation test for starch. Fermentation test was carried out by the standard Durham-tube method. ${ }^{1)}$ Cells were cultured in a test tube $(1.8 \times 18 \mathrm{~cm})$ containing $5 \mathrm{ml}$ of YPS medium at $28^{\circ} \mathrm{C}$ for up to 3 weeks.

Flocculation test. Cells were cultured with shaking in a test tube $(1.5 \times 10.5 \mathrm{~cm})$ containing $1 \mathrm{ml}$ of YEPD medium at $28^{\circ} \mathrm{C}$ for $1 \sim 2$ days. Flocculating cultures were easily distinguished from non-flocculating ones because the former produced a sediment of cells.

Protoplast fusion. Fusion of protoplasts was carried out with selection for prototrophy as described previously. ${ }^{4}$

Assay for glucoamylase activity. Each reaction mixture (final volume, $0.5 \mathrm{ml}$ ) contained $0.4 \%$ soluble starch, $80 \mathrm{mM}$ sodium acetate buffer ( $\mathrm{pH} 5.0$ ), and an enzyme solution. The reaction mixture was incubated at $50^{\circ} \mathrm{C}$ for $20 \mathrm{~min}$, and the reducing sugar (glucose) formed was determined by the standard Somogyi-Nelson method. ${ }^{2)}$ Under the condition used here, the activity proceeded linearly for at least $30 \mathrm{~min}$.

Genetic analysis. Genetic techniques including tetrad analysis were described previously. ${ }^{4}$ )

\section{RESULTS AND DISCUSSION}

When cells of $S$. diastaticus were cultured on a YPSB plate at $28^{\circ} \mathrm{C}$ for $4 \sim 6$ days and then 
TABLE I. S. diastaticus STRaINS

\begin{tabular}{lll}
\hline \multicolumn{1}{c}{ Strain } & \multicolumn{1}{c}{ Genotype $^{a}$} & \multicolumn{1}{c}{ Source } \\
\hline YIY2-11A & a leu2-3,112 his4 STA1 FLO8 & This work \\
YIY2-12D & $\begin{array}{l}\text { a leu2-3,112 his2 lys2 STA1 } \\
\text { FLO8 }\end{array}$ & This work \\
YIY11-1B & a leu2-3,112 STA1 FLO8 & This work \\
YIY4-3A & $\alpha$ his4 arg4 STA1 FLO8 & This work \\
YIY256 & a arg4 STA1 FLO8 & This work \\
YIYF & a leu2-3,112 his4 sta FLO8 & This work \\
\hline
\end{tabular}

a Recently, we found that $S$. cerevisiae carries an inhibitor gene (INH 1) against glucoamylase production, and then the genotype of $S$. diastaticus is proposed to be $S T A 1 i n h^{\circ}$. Details will be published elsewhere (manuscript submitted).

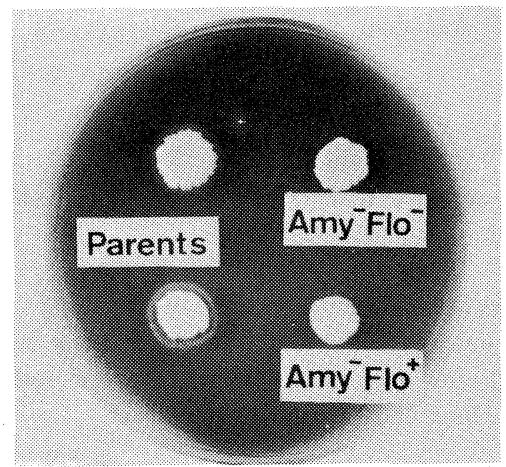

FIG. 1. Halo Formation on the YPSB Plate.

The parent strains (top, YIY11-1B; bottom, YIY2-11A) formed halos around colonies. The Amy ${ }^{-}$mutant strains (top, YKF12; bottom, YKF2) formed no halo.

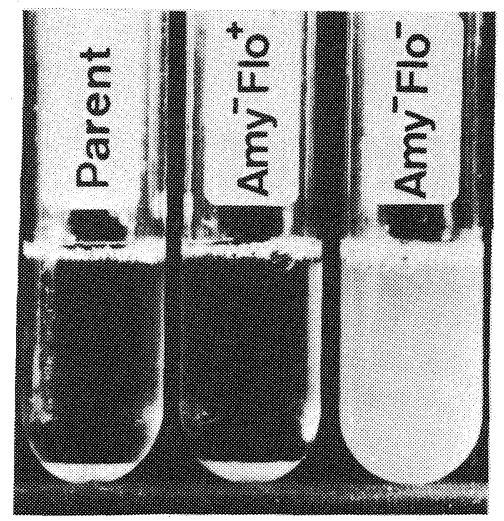

FIG. 2. Flocculation in YEPD medium.

The parent strain YIY11-1B (left) and the $\mathrm{Amy}^{-} \mathrm{Flo}^{+}$ mutant strain YKF2 (center) were highly flocculent and cells were immediately sedimented to the bottom of the test tubes. The Amy ${ }^{-} \mathrm{Flo}^{-}$mutants strain YKF12 (right) were non-flocculent.
Table II. Glucoamylase ACtivity AND THE OTHER PHENOTYPES OF THE Parent and the Mutant Strains

\begin{tabular}{|c|c|c|c|c|}
\hline Strain & $\begin{array}{c}\text { Glucoamylase } \\
\text { activity }^{a}\end{array}$ & $\mathrm{Halo}^{b}$ & $\begin{array}{l}\text { Fermen- } \\
\text { tation }^{c}\end{array}$ & $\begin{array}{l}\text { Floccu- } \\
\text { lation }^{d}\end{array}$ \\
\hline \multicolumn{5}{|l|}{ Parent } \\
\hline YIY2-11A & 16.3 & + & + & + \\
\hline YIY2-12H & 16.3 & + & + & + \\
\hline YIY11-1B & 15.0 & + & + & + \\
\hline \multicolumn{5}{|l|}{ Mutant } \\
\hline YKF2 & 0.6 & - & - & + \\
\hline YKF5 & 0.5 & - & - & + \\
\hline YKF12 & 0.6 & - & - & - \\
\hline YKF13 & 0.7 & - & - & - \\
\hline
\end{tabular}

a Cells were cultured in YPS medium at $28^{\circ} \mathrm{C}$ for 3 days. The culture supernatants were obtained by centrifugation, dialyzed extensively against deionized water, and assayed for glucoamylase activity. The activity is presented as increased absorbance at $660 \mathrm{~nm}$ for $20 \mathrm{~min}$ per $0.1 \mathrm{ml}$ of enzyme solution.

${ }^{b}$ A small amount of cells were smeared on the YPSB plate, and the plate was incubated successively at $28^{\circ} \mathrm{C}$ for 6 days and at $4^{\circ} \mathrm{C}$ for 2 days. + , haloforming; - , halo-non-forming.

${ }^{c}+$, fermentable; - , non-fermentable.

${ }^{d}+$, flocculating; - , non-flocculating.

plate was incubated at $4^{\circ} \mathrm{C}$ for $1 \sim 2$ days, a halo was formed around each colony (Fig. 1). The halo formation reflected the glucoamylase activity, since when a series of diluted enzyme solution were incubated in wells in a YPSB plate at $50^{\circ} \mathrm{C}$ (an optimal temperature for the enzyme activity) for $1 \mathrm{hr}$, followed by incubation at $4{ }^{\circ} \mathrm{C}$ overnight, halos were formed around the wells with the size of the halo increasing with the increasing activity present in the wells. Thus, in tetrad analysis, we scored glucoamylase-producing ability (producing, $\mathrm{Amy}^{+}$; non-producing, $\mathrm{Amy}^{-}$) by means of halo formation on YPSB plates. This method has allowed us to determine glucoamylaseproducing ability of the large number of cultures necessary for genetic studies. The tetrad data were consistent with those obtained from a starch fermentation test by a classical Durhamtube method. ${ }^{1)}$

Glucoamylase-non-producing $\left(\mathrm{Amy}^{-}\right) \mathrm{mu}-$ tants were isolated from three wild-type strains 
YIY2-11A, YIY2-12D, and YIY11-1B (Table I), after mutagenesis with ethylmethane sulfonate (final concentration, 3\%). Mutagenized cells (survival, $50 \%$ ) were spread on the YPSB plates, followed by incubation at $28^{\circ} \mathrm{C}$ for 6 days and at $4{ }^{\circ} \mathrm{C}$ for 2 days. Colonies forming no halo were picked up and subcultured to single colonies on the YPSB plate. We obtained 146 independent mutants. They showed no starch fermentation. Fifty-eight of the mutants were highly flocculent $\left(\mathrm{Flo}^{+}\right)$, however, the remaining 88 mutants were non-flocculent $\left(\mathrm{Flo}^{-}\right)$. Four mutant strains were chosen for further analysis: YKF2 and YKF5 are Amy ${ }^{-}$ $\mathrm{Flo}^{+}$, and YKF12 and YKF13 are Amy ${ }^{-}$ Flo $^{-}$(Figs. 1 and 2). The mutant strains YKF2 and YKF5 were derived from the parent strains YIY2-12D and YIY11-1B, respectively, and the mutant strains YKF12 and YKF13 were derived from the parent strain YIY211A. Table II summarizes glucoamylase activity secreted and the other phenotypes of the parent and the mutant strains. All mutant strains produced greatly diminished glucoamylase acrivity. These mutants were crossed with a wild-type strain YIY4-3A, and the resulting diploids were subjected to tetrad analysis after sporulation, of which the results are presented in Table III. The tetrad data suggest that the mutant phenotypes, i.e. $\mathrm{Amy}^{-} \mathrm{Flo}^{+}$ and $\mathrm{Amy}^{-} \mathrm{Flo}^{-}$, are caused by single muta- tions. Determination of dominant or recessive phenotype of the mutations and their complementation tests were carried out in strains homozygous for a mating type allele $(M A T)$, since the mating type constitution $M A T a /$ $M A T \alpha$ represses glucoamylase production. Details will be published elsewhere (manuscript No. 3-355). Strains homozygous for $M A T$ were constructed by a standard protoplast fusion method ${ }^{4)}$ with selection for prototrophy. Most of the fusants are expected to be diploids, since, in the fusion conditions used, all six fusants examined were diploids, as estimated by tetrad analysis using a standard diploid strain homozygous for $M A T$. To determine dominant or recessive phenotype of the mutations, fusants were constructed using two haploid strains carrying MATa: one was a wild-type strain, YIY256, and the other was one of the mutant strains. Table IV shows the following abilities of the fusant strains: glucoamylase activity, halo formation, starch fermentation, and flocculation. The phenotypes of all fusant strains heterozygous for the mutant alleles were converted to the wild type, indicating that all mutations are recessive. Complementation tests were carried out in fusant strains constructed by combination of two haploid mutant strains carrying MATa. Table $\mathrm{V}$ shows the following abilities of the fusant strains: glucoamylase activity, halo for-

Table III. Tetrad Analysis in the Cross Between the Wild-type and THE Mutant STRains

\begin{tabular}{ccc}
\hline Cross & Segregation & No. of asci \\
\hline YIY4-3A $\times$ YKF2 & $2\left(\mathrm{Amy}^{+} \mathrm{Flo}^{+}\right): 2\left(\mathrm{Amy}^{-} \mathrm{Flo}^{+}\right)$ & 24 \\
YIY4-3A $\times$ YKF5 $_{\text {YIY4-3A } \times \text { YKF12 }}$ & $2\left(\mathrm{Amy}^{+} \mathrm{Flo}^{+}\right): 2\left(\mathrm{Amy}^{-} \mathrm{Flo}^{+}\right)$ & 24 \\
YIY4-3A $\times$ YKF13 $^{-}$ & $2\left(\mathrm{Amy}^{+} \mathrm{Flo}^{+}\right): 2\left(\mathrm{Amy}^{-} \mathrm{Flo}^{-}\right)$ & 12 \\
\hline
\end{tabular}

Table IV. Recessive Phenotype of the Mutations

\begin{tabular}{ccccc}
\hline Fusant & $\begin{array}{c}\text { Glucoamylase } \\
\text { activity }^{a}\end{array}$ & Halo $^{b}$ & Fermentation $^{c}$ & Flocculation $^{d}$ \\
\hline YIY256 $\times$ YKF2 & 4.3 & + & + & + \\
YIY256 $\times$ YKF5 & 5.6 & + & + & + \\
YIY256 $\times$ YKF12 & 9.2 & + & + & + \\
YIY256 $\times$ YKF13 & 11.4 & + & + & + \\
\hline
\end{tabular}

a S See Table II (footnotes $a \sim d$, respectively). 
TABle V. Complementation Test

\begin{tabular}{|c|c|c|c|c|c|c|c|c|c|c|c|c|c|c|c|c|}
\hline \multirow{2}{*}{ Mutant $^{a}$} & \multicolumn{4}{|c|}{ YKF2 } & \multicolumn{4}{|c|}{ YKF5 } & \multicolumn{4}{|c|}{ YKF12 } & \multicolumn{4}{|c|}{ YKF13 } \\
\hline & A & B & $\mathrm{C}$ & D & A & B & $\mathrm{C}$ & D & A & B & $\mathrm{C}$ & D & A & B & $\mathrm{C}$ & D \\
\hline YKF2-1 & 0.6 & - & - & + & 0.5 & - & - & + & 3.0 & + & + & + & 2.7 & + & + & + \\
\hline YKF5-1 & & & & & 0.9 & - & - & + & 3.6 & + & + & + & 3.6 & + & + & + \\
\hline YKF12-1 & & & & & & & & & 0.6 & - & - & - & 0.7 & - & - & - \\
\hline YKF13-1 & & & & & & & & & & & & & 0.7 & - & - & - \\
\hline
\end{tabular}

a Mutant strains YKF2-1, YKF5-1, YKF12-1, and YKF13-1 were derived from the crosses between the wild-type strains YIY4-3A and each of the original mutant strains YKF2, YKF5, YKF12, and YKF13.

Lane A, glucoamylase activity; Lane B, halo formation; Lane C, fermentation; and Lane D, flocculation. See footnotes in Table II.

Table VI. Tetrad Analysis in the Cross between the $s a^{\circ}$ Strain AND the amy Mutant Strains

\begin{tabular}{|c|c|c|c|c|}
\hline \multicolumn{2}{|c|}{ Cross } & \multicolumn{3}{|c|}{ No. of $\operatorname{asci}^{b}$} \\
\hline Genotype & Strain $^{a}$ & $0^{+}: 4^{-}$ & $2^{+}: 2^{-}$ & $1^{+}: 3^{-}$ \\
\hline$s t a^{\circ} \times a m y 1-1$ & $\mathrm{YIYF} \times \mathrm{YKF} 2-2$ & 18 & 0 & 0 \\
\hline sta $^{\circ} \times a m y 1-2$ & YIYF $\times$ YKF5-2 & 18 & 0 & 0 \\
\hline $\operatorname{sta}^{\circ} \times a m y 2-1$ & YIYF $\times$ YKF12-2 & 1 & 2 & 7 \\
\hline sta ${ }^{\circ} \times a m y 2-2$ & YIYF $\times$ YKF13-2 & 3 & 2 & 7 \\
\hline
\end{tabular}

a Mutant strains YKF2-2, YKF5-2, YKF12-2, and YKF13-2 were derived from the crosses between the wild-type strain YIY4-3A and each of the original mutant strains YKF2, YKF5, YKF12, and YKF13.

${ }^{b}$ Amy phenotype was determined by means of halo formation as described in Table II, footnote $b$.

mation, starch fermentation and flocculation. The results indicate that the four mutant alleles are divided into two complementation groups: the mutant alleles present in the mutant strains YKF2 and YKF5 were designated amy $1-1$ and $a m y 1-2$, respectively, and the other alleles present in the mutant strains YKF12 and YKF13 were designated amy2-1 and amy2-2, respectively.

A strain YIYF is one of the glucoamylasenon-producing segregants derived from the cross between two strains of $S$. diastaticus: one carrying the $S T A 1$ gene and the other carrying a $S T A 3$ gene. In asci from diploids constructed by the crosses between the strain YIYF and $S$. diastaticus strains carrying either STA1 or $S T A 3$, glucoamylase-producing ability is segregated as 2(producing): 2 (non-producing), suggesting that the strain YIYF carries no active glucoamylase-producing genes, so it is designated $s t a^{\circ}$. Mutant strains carrying each of the four mutant alleles amy1-1, amy1-2, amy2-1, and amy2-2, were crossed with the strain YIYF and the resulting diploids were subjected to tetrad analysis after sporulation. The results are presented in Table VI. In the crosses between the strain YIYF and the amy1 mutant strains, all segregants were Amy-, suggesting that the amyl mutation may be located in the $S T A 1$ gene, which may be the structural gene for glucoamylase I. In the crosses between the strain YIYF and the amy2 mutant strains, three different ascus types appeared, suggesting that the amy 2 mutant strains carry the intact $S T A 1$ gene, and the product of the $A M Y 2$ gene was simultaneously involved in both production of glucoamylase I and flocculation governed by the FLO8 gene, since the amy 2 mutation made yeast cells $\mathrm{Amy}^{-} \mathrm{Flo}^{-}$.

Acknowledgments. We are grateful to H. Tamaki for providing $S$. diastaticus strains. We also thank $\mathbf{K}$. Fukuhara for technical assistance. This investigation was 
supported in part by a Grant-in-Aid for Scientific Research (Grant No. 57211018) from the Ministry of Education, Science and Culture of Japan.

\section{REFERENCES}

1) H. Tamaki, Mol. Gen. Genet., 164, 205 (1978).

2) H. Tamaki, Ann. Rep. Studies, Doshisha Women's
College, 31, 166 (1980).

3) I. Yamashita, T. Maruyama, K. Fukuhara, K. Suzuki, T. Maemura and S. Fukui, Abstracts of Papers, Annual Meeting of Agric. Chem. Soc. Japan, 1983 , p. 352.

4) T. Maemura, I. Yamashita and S. Fukui, FEBS Lett., 158, 50 (1983). 